\title{
Big Data Revolution in Health Care Sector
}

\author{
Gagana $S^{*}$, Paramesha K \\ Dept. of Computer Science and Engineering, Vidya Vardhaka College \\ of Engineering Mysuru, India
}

DOI: https://doi.org/10.21467/proceedings.1.44

* Corresponding author email: gaganashivaraju20@gmail.com

\begin{abstract}
Health care sector grows tremendously in last few decades. The health care sector has generated huge amounts of data that has huge volume, enormous velocity and vast variety. Also, it comes from a variety of new sources as hospitals are now tend to implemented electronic health record (EHR) systems. These sources have strained the existing capabilities of existing conventional relational database management systems. In such scenario, big data solutions offer to harness these massive, heterogeneous and complex data sets to obtain more meaningful and knowledgeable information. However, the adoption rate and research development in this space is still hindered by some fundamental problems inherent within the big data paradigm. Recent research that targets utilization of large volumes of medical data while combining multimodal data from disparate sources is discussed. Potential areas of research within this field which have the ability to provide meaningful impact on healthcare delivery are also examined. Keywords-big data, health care, hadoop.
\end{abstract}

\section{INTRODUCTION}

The term "big data" has become a buzzword in recent years. Big data in health is concerned with meaningful datasets that are too big, too fast, and too complex for healthcare providers to process and interpret with existing tools. It is driven by continuing effort in making health services more efficient and sustainable given the demands of a constantly expanding population with an inverted age pyramid, as well as the paradigm shift of delivering health services toward prevention, early intervention and optimal management [2]. Despite the inherent complexities of healthcare data, there is potential and benefit in developing and implementing big data solutions within this realm [4].

Historical approaches to medical research have generally focused on the investigation of disease states based on the changes in physiology in the form of a confined view of certain singular modality of data [6]. Big data in healthcare is overwhelming not only because of its volume but also because of the diversity of data types and the speed at which it must be managed. The totality of data related to patient healthcare and wellbeing make up "big data" in the healthcare industry [5]. By discovering associations and understanding patterns and trends within the data, big data analytics has the potential to improve care, save lives and lower costs. Thus, big data analytics applications in healthcare take advantage of the

\footnotetext{
(C) 2018 Copyright held by the author(s). Published by AIJR Publisher in Proceedings of the $3^{\text {rd }}$ National Conference on Image Processing, Computing, Communication, Networking and Data Analytics (NCICCNDA 2018), April 28, 2018.

This is an open access article under Creative Commons Attribution-NonCommercial 4.0 International (CC BY-NC 4.0) license, which permits any non-commercial use, distribution, adaptation, and reproduction in any medium, as long as the AijR license, which permits any non-commercial use, distribution
} 
Big Data Revolution in Health Care Sector

explosion in data to extract insights for making better informed decisions [7]. Big Data technology and services market represents a fast-growing multibillion-dollar worldwide opportunity in the field of Health care sector to provide meaningful impact.

\section{SYSTEM ARCHITECTURE}

The conceptual framework for a big data analytics project in healthcare is similar to that of a traditional health informatics or analytics project. The key difference lies in how processing is executed. In a regular health analytics project, the analysis can be performed with a business intelligence tool installed on a stand-alone system, such as a desktop or laptop. Because big data is by definition large, processing is broken down and executed across multiple nodes. The concept of distributed processing has existed for decades. What is relatively new is its use in analyzing very large data sets as healthcare providers start to tap into their large data repositories to gain insight for making better-informed health-related decisions. Furthermore, open source platforms such as Hadoop / MapReduce, available on the cloud, have encouraged the application of big data analytics in healthcare. Figure 1 represents, while the algorithms and models are similar, the user interfaces of traditional analytics tools and those used for big data are entirely different; traditional health analytics tools have become very user friendly and transparent. Big data analytics tools, on the other hand, are extremely complex, programming intensive, and require the application of a variety of skills. They have emerged in an ad hoc fashion mostly as open-source development tools and platforms, and therefore they lack the support and user-friendliness that vendor-driven proprietary

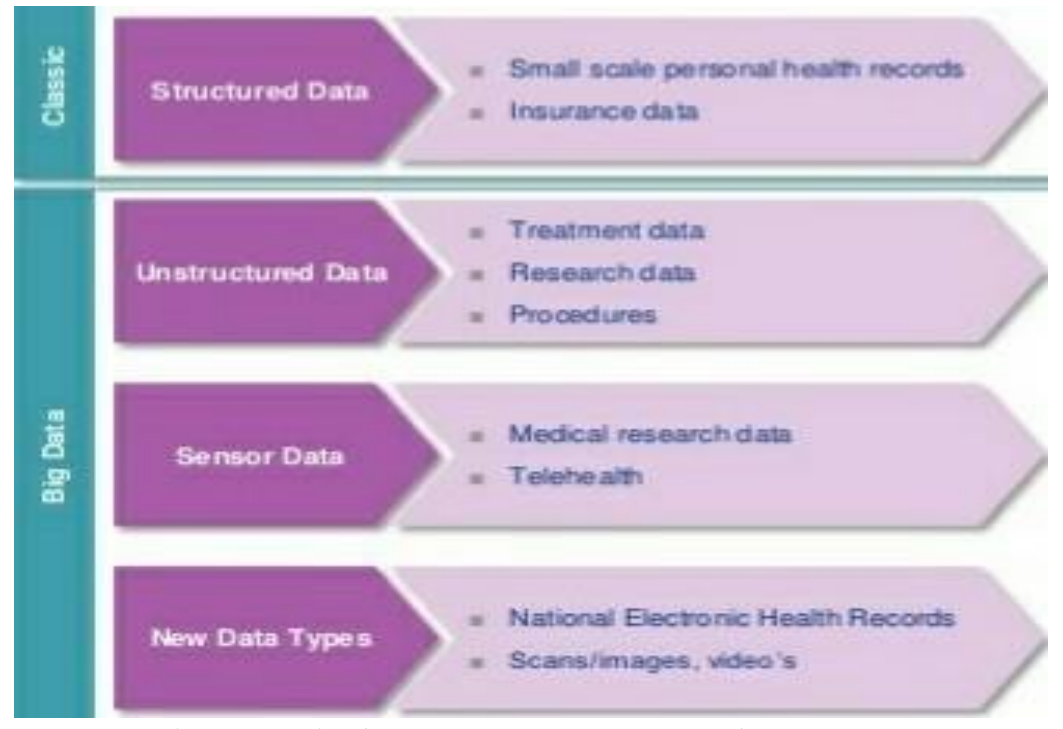

Figure 1: Big data case components tools possess.

Figure 2 represents, Big data in healthcare can come from internal (e.g., electronic health records, clinical decision support systems, CPOE, etc.) and external sources (government sources, laboratories, pharmacies, insurance companies \& HMOs, etc.), often in multiple formats and residing at multiple locations (geographic as well as in different healthcare 
providers' sites) in numerous legacy and other applications (transaction processing applications, databases, etc.
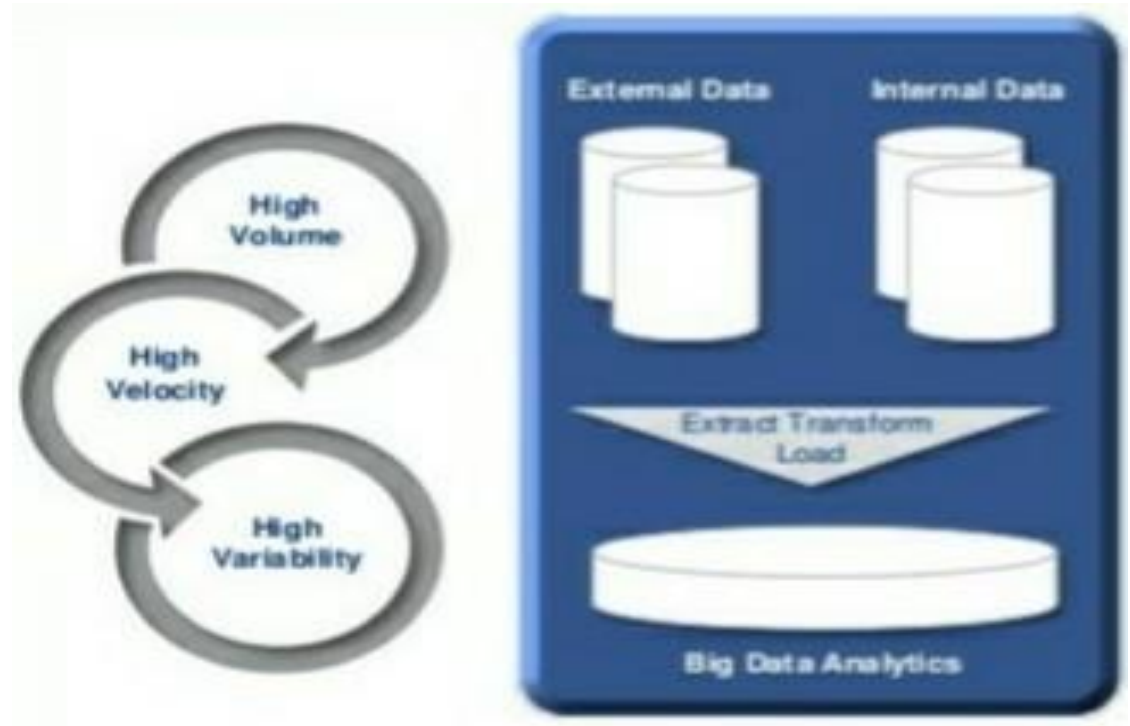

Figure 2: Big data architecture

\section{BIG DATA IN HEALTH CARE KEY COMPONENTS}

In Step 1, the interdisciplinary big data analytics in healthcare team develops a 'concept statement'. This is a first cut at establishing the need for such a project. The concept statement is followed by a description of the project's significance. The healthcare organization will note that there are trade-offs in terms of alternative options, cost, scalability, etc. Once the concept statement is approved, the team can proceed to Step 2, the proposal development stage. Here, more details are filled in. Based on the concept statement, several questions are addressed: What problem is being addressed? Why is it important and interesting to the healthcare provider? What is the case for a 'big data' analytics approach? The project team also should provide background information on the problem domain as well as prior projects and research done in this domain.

Next, in Step 3, the steps in the methodology are fleshed out and implemented. The concept statement is broken down into a series of propositions. Simultaneously, the independent and dependent variables or indicators are identified. The data sources are also identified; the data is collected, described, and transformed in preparation for analytics. A very important step at this point is platform/tool evaluation and selection. There are several options available, as indicated previously, including AWS Hadoop, Cloudera, and IBM BigInsights. The next step is to apply the various big data analytics techniques to the data. This process differs from routine analytics only in that the techniques are scaled up to large data sets. Through a series

Proceedings of the $3^{\text {rd }}$ National Conference on Image Processing, Computing, Communication, Networking and Data Analytics (NCICCNDA 2018) 
Big Data Revolution in Health Care Sector

of iterations and what-if analyses, insight is gained from the big data analytics. From the insight, informed decisions can be made.

In Step 4, the models and their findings are tested and validated and presented to stakeholders for action. Figure 3 represents, Implementation is a staged approach with feedback loops built in at each stage to minimize risk of failure.
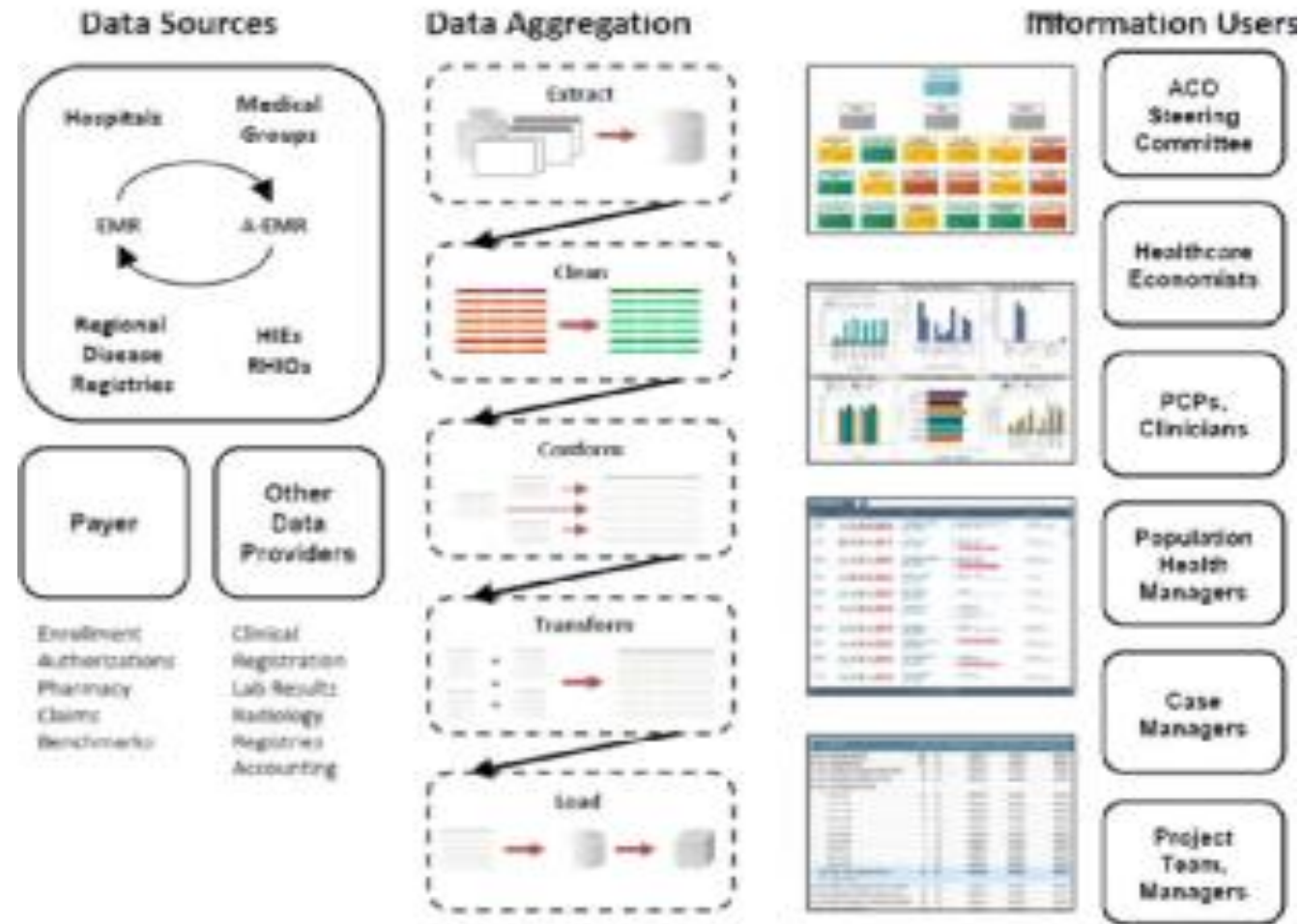

Figure 3: Big data in health care key components

\section{BIG DATA FOR REAL WORLD CLINICAL ANALYTICS}

Client is a leading diagnostic services provider that assists patients and physicians in making better healthcare decisions to improve patient outcomes. In order to enhance the quality of its analytics and decision support systems, client wanted to replace their legacy data warehousing solution with a data lake that could manage high volumes of data (5-10 million messages per day) and provide deeper interactive visualizations (historical and longitudinal reporting, trend analysis, etc.) and analytical queries on massive healthcare datasets.

Figure 4 represents, the solution is based on Cloudera Hadoop distribution, MapReduce, Spark Streaming and other Hadoop/ Big data technologies. The solution also included building a flexible and configurable data model to represent healthcare data, and enable advanced analytics. 


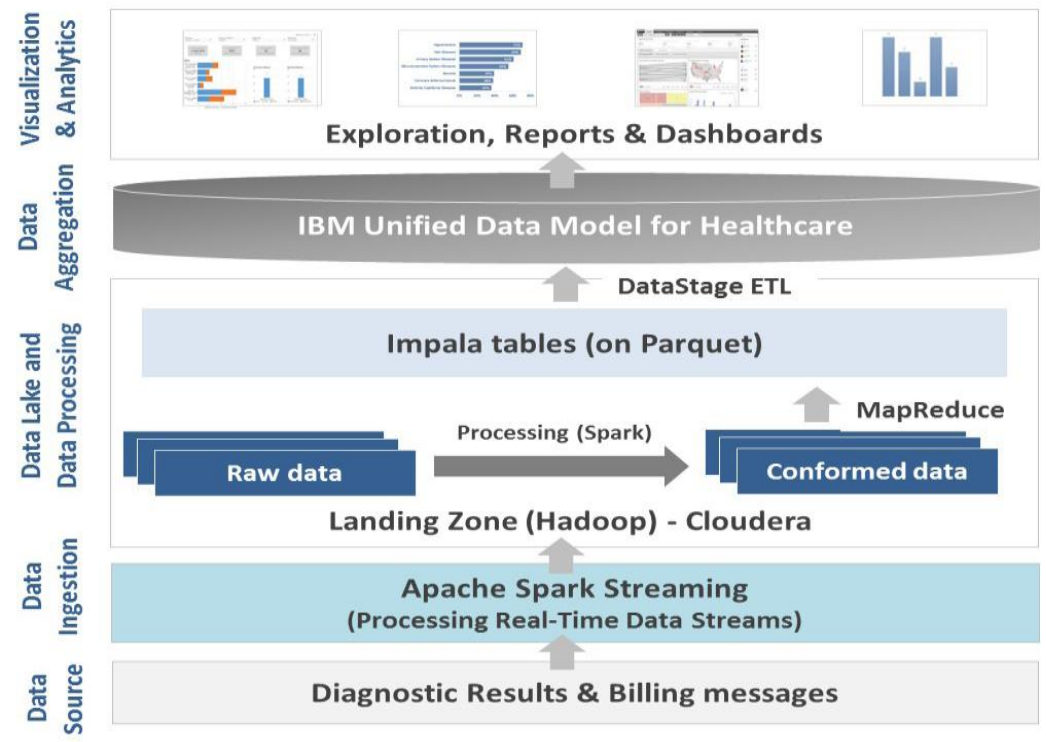

Figure 4: Big Data for real world clinical analytics

\section{NEED OF HADOOP IN HEALTH CARE DATA SOLUTIONS}

\subsection{Hadoop technology in Cancer Treatments and Genomics}

The biggest reason why cancer has not been cured yet is because of the fact that cancer mutates in different patterns and reacts in different ways based on the genetic makeup of an individual. Leveraging Hadoop technology will offer great support for parallelization and help in mapping the 3 billion DNA base pairs using MapReduce programs.

\subsection{Hadoop technology in Monitoring Patient Vitals}

There are several hospitals across the world that uses Hadoop to help the hospital staff work efficiently with Big Data. Without Hadoop, most patient care systems could not even imagine working with unstructured data for analysis.

\subsection{Hadoop technology in the Hospital Network}

A Cleveland Clinic spinoff company known as Explorys is making use of Big Data in healthcare to provide the best clinical support, reduce the cost of care measurement and manage the population of at-risk patients. Explorys uses Hadoop technology to help their medical experts analyze data bombardments in real time from diverse sources such as financial data, payroll data, and electronic health records.

\subsection{Hadoop technology in Healthcare Intelligence}

Using Hadoop technology in Healthcare Intelligence applications helps hospitals, payers and healthcare agencies increase their competitive advantages by devising smart business solutions.

Proceedings of the $3^{\text {rd }}$ National Conference on Image Processing, Computing, Communication, Networking and Data Analytics (NCICCNDA 2018) 
Big Data Revolution in Health Care Sector

5.5 Hadoop technology in Fraud Prevention and Detection

Big Data Analytics helps healthcare insurance companies find different ways to identify and prevent fraud at an early stage. Using Hadoop technology, insurance companies have been successful in developing predictive models to identify fraudsters by making use of real-time and historical data of medical claims, weather data, wages, voice recordings, demographics, cost of attorneys and call center notes.

\section{OPPORTUNITY OF BIG DATA IN HEALTH CARE}

\subsection{Decreasing Healthcare Costs to Get Financial Profit}

Big data can help decrease the cost of providing medical treatment in many ways. Moreover, analysis on data gives insight to health care providers to determine populations at risk for illness. By doing so, proactive steps can be taken initially. Big Data can more accurately pinpoint where education and prevention is needed to produce healthier population at low costs. Treatment is more evidence based using Big Data.

\subsection{Promotes Research and Innovation}

By analytics on data, the current state of health of patients provides insight to them to take more ownership of their healthcare. The information sharing mechanism increases productivity and reducing overlapping of data. By thus, it is enhancing the coordination of care [4].

\subsection{Personalized Medicine}

In past few years, it is possible to predict the lifestyle diseases through genetic blue prints. Big data will further personalize medicine by determining the tests and treatments needed for each patient. The provision of earlier treatment can reduce the health costs and can eliminate the risk of chronic diseases [4].

\subsection{Strengthen the Preventive Care}

Prevention is always better than cure. Following this thumb of rule, with the advent of Big Data analytics, it is easy to capture, analyze and compare patient symptoms earlier to offer a preventive care in a better way.

\subsection{Virtual Care and Wearable Health Care Technologies}

Technology is helping providers make virtual care initiatives that increase quality of care and provide patients with more access [3].

\subsection{Health Trend Analysis}

By using different analytical approaches including data mining and text mining techniques, health trend analysis and comprehensive patient management is easier using Big Data Analytics [7].

\subsection{Identification and Tracking of Patients}

The identification and tracking of patients with type 2 diabetes is discussed in recent article [6]. The author suggests using a two-step process to identify subsets of patients that have 
similar clinical indications and care patterns. In a first step, patients are divided into groups based on the primary diagnosis. Then after, a statistical clustering method is applied to further divide the subsets. This method uses readily available administrative datasets. Also, patients must be tracked longitudinally to determine the patterns for treatment. Therefore, the method is applicable in scenarios where patient data is available over time and across providers [16].

\subsection{Studying Drug Efficiency}

Electronic health record (EHR) data may also be used to study drug efficiency. It has been observed that the cost of randomized controlled trials is much higher than the cost of using readily available EHR data to compare treatment modalities [16].

\section{CHALLENGES OF BIG DATA IN HEALTH CARE}

\subsection{Protecting the Patient's Privacy}

One of the significant challenges in leveraging health care's big data to its full extent is policies that protect the privacy of patient's data. Many laws protect the patient's data and not reveal the patient's identity that makes the big data analytics difficult.

\subsection{Data Aggregation}

In health care sector, the data is unstructured. These unstructured data are in the form of images, graphs, notes of doctor's etc. These may lead a huge problem at the time of aggregation of these data. Natural language processing and free text software could solve this problem at some extend but it is in its initial stage.

\subsection{Cost Incurred for Establishment of Big Data Architecture}

To have a benefit through big data analytics, it requires organization level management and analysis as well as a large scale investment.

\subsection{Requirement of Expert Knowledge}

Big data system requires data scientists with specialized experience to support design, implementation and continued use. Data scientists need highly technical skill sets [22]. They must possess soft skills such as communication, collaboration, leadership, creativity and more.

\subsection{Security Concern}

Health data is a personal data. Patient expects extra privacy protection if they are going to fully practice in Big Data Analytics project. In such types of project, user should be authorized at different levels and time periods. These will prevent unauthorized access to medical records.

\section{STATISTICS OF BIG DATA IN HEALTH CARE SECTOR}

Figure 5 represents the growth of Big Data in health care sector within a range of 2012-2020.

Proceedings of the $3^{\text {rd }}$ National Conference on Image Processing, Computing, Communication, Networking and Data Analytics (NCICCNDA 2018) 
Expected growth in

healthcare data,

2012-2020 (petabytes)

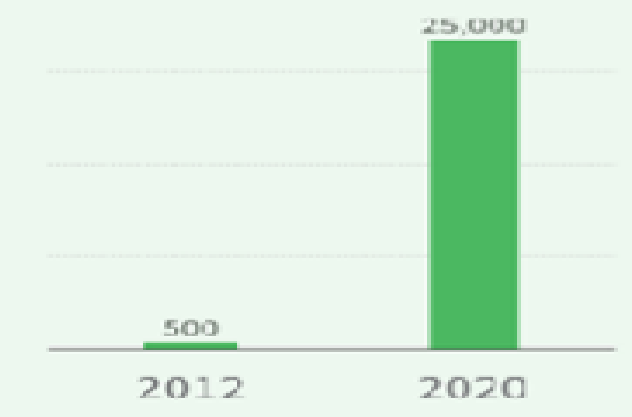

Figure 5: Statistics of Big Data in health care sector

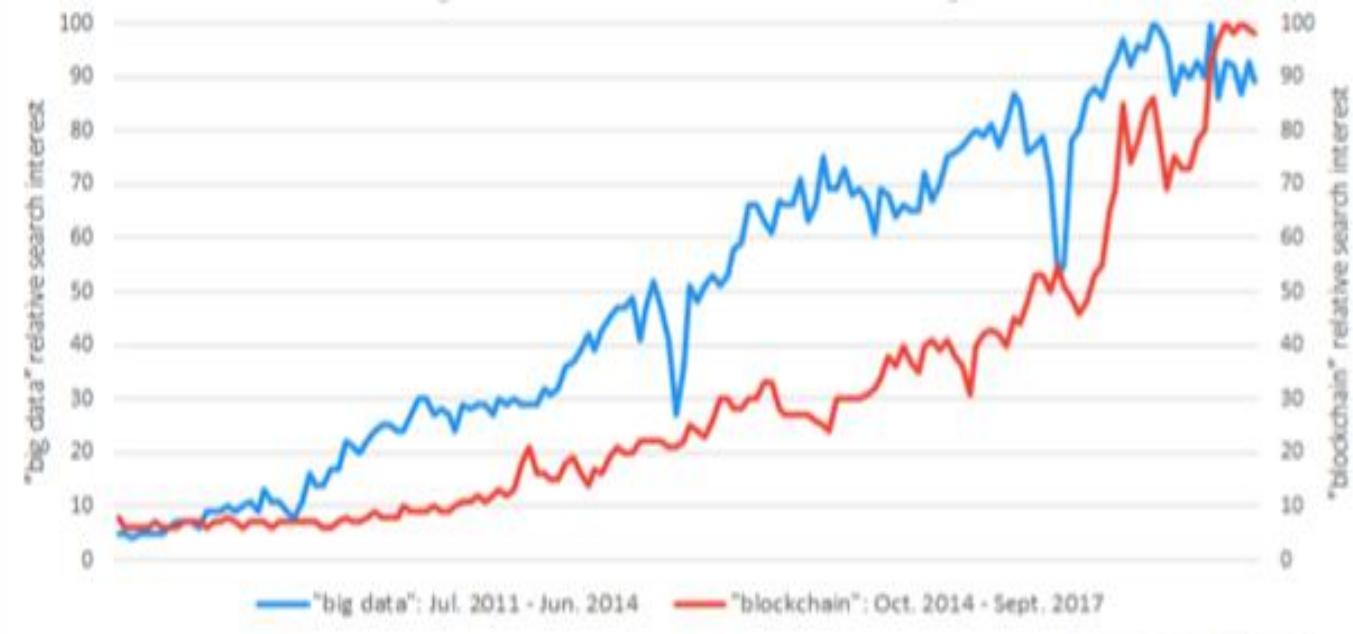

Figure 6: Block chain v/s Big Data

Figure 6 represents the comparison of interest in Big Data and Block chain over the course of 3 years. The hype around Block chain is much more abrupt compared to the gradual interest increase in Big Data during 2011-2014. 


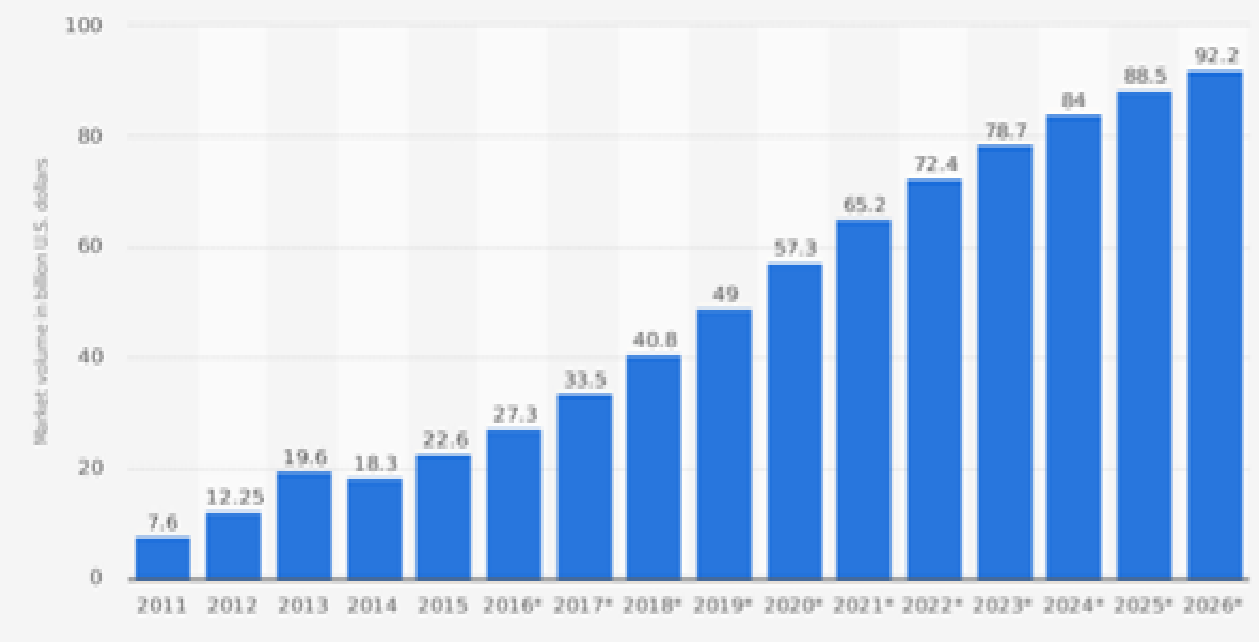

Figure 7: Big Data market size revenue forecast

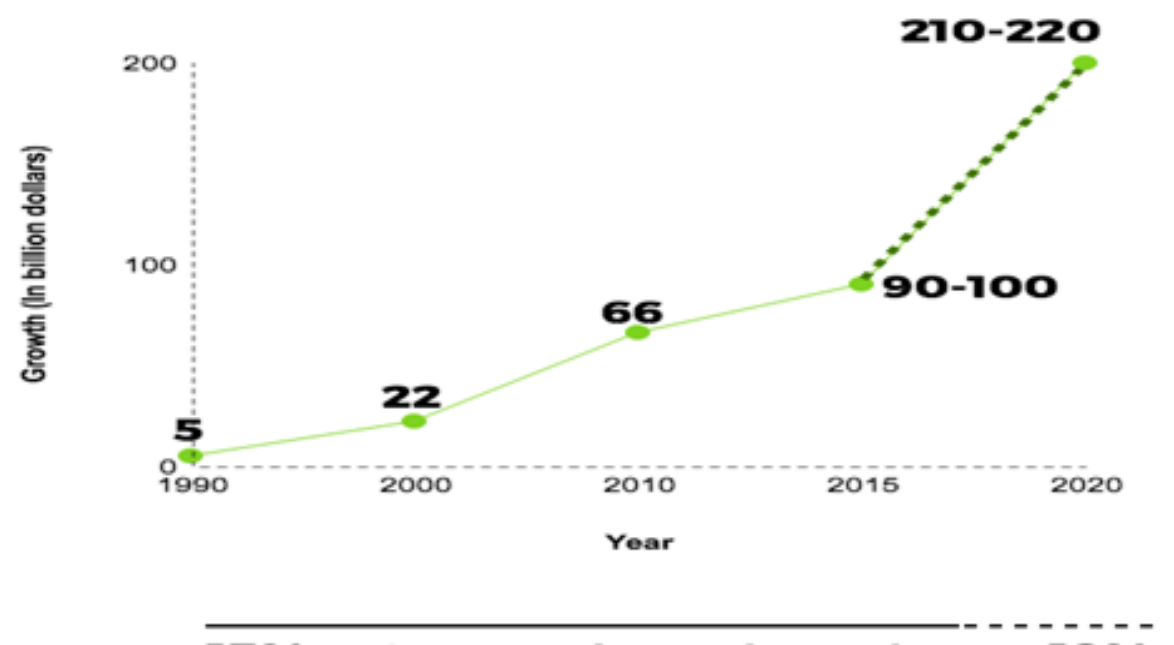

$13 \%$

Compound annual growth rate $18 \%$

Figure 8: Health Sector growth trend

Figure 7 represents the Big Data market size revenue worldwide from 2011-2016. Figure 8 represents the growth in health care sector by making use of Big Data in the processing stage.

\section{HEALTH CARE SECTOR WITHOUT BIG DATA}

Most health system can do plenty today without big data, including meeting most of their analytics and reporting needs. We haven't even come close to stretching the limits of what health care analytics can accomplish with traditional relational databases and using these databases effectively is a more valuable focus than worrying about big data [25]. Currently, majority of health care institutions are swamped with some very pedestrian problems such as regulatory reporting and operational dashboards. Most just need the proverbial "air and water"

Proceedings of the $3^{\text {rd }}$ National Conference on Image Processing, Computing, Communication, Networking and Data Analytics (NCICCNDA 2018) 
Big Data Revolution in Health Care Sector

right now, but once basic needs are met and some of the initial advanced application are in place, new use cases will arrive driving the need for big data solutions.

\section{FUTURE OF HEALTH CARE AND TRANSITION TO BIG DATA}

Today, health system's need for data driven quality and cost improvement is urgent. Health care organizations cannot afford to wait for big data technology to mature before dividing into analytics [18]. The important factor will be choosing a data warehousing solution that can easily adapt to the future of big data. A Late-Binding Enterprise Data warehouse (EDW) architecture is ideal for making the transition from relational databases to unstructured big data. The late binding approach, in fact, very similar to big data approach. In a Late Binding EDW like health catalysts, data from source systems are stored into source marts.

\section{SOCIAL MEDIA IN HEALTH CARE SECTOR}

Social media in the public and private sector has expanded exponentially. Consumer groups and professional sites have increased direct accessibility between groups and individuals. Direct engagement between public and private sectors creates opportunities to develop solutions quickly and efficiently. Various businesses and government agencies have taken advantage of social media and the resulting impact of their investments. The return on investments ranged from improving customer service to improving communications with the public. In 2009, the Centers for Disease Control and Prevention (CDC) took advantage of social media posts made by users ranging from possible symptoms to claims of possible outbreak of the H1N1 virus. In addition to traditional avenues, the CDC used social media to directly engage the public. The public and the federal government benefitted from this approach. The general public quickly acquired information to make decisions such as identifying symptoms and locations to avoid due to a possible outbreak. The government intelligently mobilized resources and prevented mass panic. A 24-hour informational hotline was created in addition to press briefings for media and health alert networks, daily postings to the CDC 2009 H1N1 website, Facebook, and Twitter, and by partnering with other organizations to reach additional audiences.

The CDC, in the wake of the pandemic, created the "Predict the Influenza Season Challenge" competition. The participants were encouraged to develop modeling tools that predicted seasonal flu activity based solely on information gathered from social media networks.

Depression, a growing global health problem, is receiving growing attention from researchers. Social media networks are allowing patients diagnosed with depression to share their thoughts and connect with other patients and doctors. Researchers argue that monitoring these networks in real-time will give health policymakers a more accurate overview of depression levels in their populations, as opposed to traditional annual surveys. The methods used to collect information include identifying (and cross-referencing) key words used in clinical depression circles and developing (and training) models to predict the user's 'mood' in a post. 
Depression, if left untreated, leads to multiple mental and physical health problems. Suicide is one risk factor for depressed patients. Researchers have been studying how to predict suicide by identifying content in social media networks that serve as 'red flags' to suicidal tendencies. These methods would contribute to a more rigorous suicide screening and prevention programs that would greatly benefit counselors and patients.

Pharmacovigilance is another can also benefit from big data mining. Adverse drug reaction (ADR) is the biggest risk factor when taking pharmaceutical drugs. Agencies have taken steps to monitor the ADRs of drugs sold in the market while developing programs to monitor social media networks to enhance their alert systems to drug side effects. These methods will allow consumers in social media networks to voice their concerns over ADRs of drugs and would demand the attention of regulatory agencies and pharmaceutical companies. The 'digital divide,' a social and economic divide that restricts access to information and communication technology, is a challenge. Another challenge may consist of the ease of access to the data, as some social media platforms allow only restricted access to user posts.

Health care organizations should be aware that while consumer feedback from social media networks can be helpful, the digital divide should also encourage them to seek other sources to confirm that information derived from offline sources matches' information from the social media networks.

\section{OPPORTUNITIES OF SOCIAL MEDIA IN HEALTH CARE SECTOR USING BIG DATA}

Social media networks, when combined with Big Data applications and health policymaking, will require a broad framework that will enable the development of smart public health applications that will result in high-quality health delivery and reduced costs. Context posting will require the development of more advanced lexicons of formal language, specific terms based on the forum topics, and development of informal dictionaries to clarify potentially confusing posts to those outside of the network. Social media platforms may require several networks that allow users multiple viewing options to ensure up-to-date information in the event that one network stops working. The large amount of data available through social media offers the promise of discovering associations; understanding patterns and trends that may help healthcare stakeholders adjust clinical path lines.

Moreover, healthcare stakeholders can use social media networks to greatly expand their communication and engagement to consumers to give the latter a greater say in product and service development, which will be a crucial precursor for the development of highlypersonalized health solutions.

The aforementioned solutions and advances can lead to further advances for post-marketing and intelligence gathering of products and services from formal (federal government) and informal (social media platforms) to respond more rapidly to consumer sentiment to remain

Proceedings of the $3^{\text {rd }}$ National Conference on Image Processing, Computing, Communication, Networking and Data Analytics (NCICCNDA 2018) 
Big Data Revolution in Health Care Sector

competitive. With the implementation of social media as part of a broader IT strategy, public health leaders, working with their international counterparts and the private sector, can proactively develop solutions that can lead to other further advances in research and development. This will be crucial as we enter the era of personalized medicine where every patient will require a unique solution based on his or her physiological and genomic data.

The investments that the private and public sectors make in social media (and IT) to engage and work with users will go a long way in improving the quality of products and services while reducing healthcare costs.

To conclude, the benefits of social media network data mining and analysis in the era of global healthcare will greatly benefit patients, national and international health agencies, and the private sector in the development and execution of smart public health policies that will ensure the higher quality of healthcare delivery at lower costs.

\section{EXAMPLES OF BIG DATA IN HEALTH CARE}

\subsection{Big Data is saving lives}

For our first example of big data in healthcare, we will look at one classic problem that any shift manager faces: how many people do I put on staff at any given time period? If you put on too many workers, you run the risk of having unnecessary labor costs add up. Too few workers, you can have poor customer service outcomes - which can be fatal for patients in industries like healthcare.

\subsection{Electronic Health Records (EHR)}

It's the most widespread application of big data in healthcare. Every patient has his own digital record which includes demographics, medical history, allergies, laboratory test results etc. Records are shared via secure information systems and are available for healthcare providers from both public and private sector. Every record is comprised of one modifiable file, which means that doctors can implement changes over time with no paperwork and no danger of data replication. EHRs can also trigger warnings and reminders when a patient should get a new lab test or track prescriptions to see if a patient has been following doctors' orders. Although EHR are a great idea, many countries still struggle to fully implement them.

\subsection{Real Time Alerting}

In hospitals, Clinical Decision Support (CDS) software analyzes medical data on the spot, providing health practitioners with advice as they make prescriptive decisions. However, doctors want patients to stay away from hospitals to avoid costly in-house treatments. Personal analytics devices, already trending as business intelligence buzzwords last year, have the potential to become part of a new healthcare delivery strategy. Wearable will collect patients' health data continuously and send this data to the cloud.

\subsection{Big Data is helping to prevent Opioid Abuse}

Our fourth example of big data analytics in healthcare is tackling a serious problem in the US. Here's a sobering fact: as of this year, overdoses from misused opioids have caused more 
accidental deaths in the U.S. than road accidents, which were previously the most common cause of accidental death. Once again, an application of big data analytics in healthcare might be the answer everyone is looking for.

\subsection{Predictive Analytics in Health Care}

We have already recognized predictive analytics as one of the biggest business intelligence trend. The goal is to help doctors make big data-informed decisions within seconds and improve patients' treatment. This is particularly useful in case of patients with complex medical histories, suffering from multiple conditions. New tools would also be able to predict, for example, who is at risk of diabetes, and thereby be advised to make use of additional screenings or weight management. To be fair, reaching out to people identified as "high risk" and preventing them from developing a drug issue is a delicate undertaking. However, this project still offers a lot of hope towards mitigating an issue which is destroying the lives of many people and costing the healthcare system a lot of money.

\subsection{Using Health Data for Informed Strategic Planning}

The use of big data in healthcare allows for strategic planning thanks to better insights into people's motivations. Care mangers can analyze check-up results among people in different demographic groups and identify what factors discourage people from taking up treatment. University of Florida made use of Google Maps and free public health data to prepare heat maps targeted at multiple issues, such as population growth and chronic diseases. Subsequently, academics compared this data with the availability of medical services in most heated areas. The insights gleaned from this allowed them to review their healthcare delivery strategy and add more care units to most problematic areas.

\subsection{Big Data in curing Cancer}

Another interesting example of the use of big data in healthcare is the Cancer Moonshot program. Before the end of his second term, President Obama came up with this program that had the goal of accomplishing 10 years' worth of progress towards curing cancer in half that time. This bold goal led to a panel which gave recommendations that included some big data use cases.

Medical researchers can use large amounts of data on treatment plans and recovery rates of cancer patients in order to find trends and treatments that have the highest rates of success in the real world. For example, researchers can examine tumor samples in bio banks that are linked up with patient treatment records. Using this data, researchers can see things like how certain mutations and cancer proteins interact with different treatments and find trends that will lead to better patient outcomes.

This data can also lead to unexpected benefits, such as finding that Desipramine, which is an anti-depressant, has the ability to help cure certain types of lung cancer.

Proceedings of the $3^{\text {rd }}$ National Conference on Image Processing, Computing, Communication, Networking and Data Analytics (NCICCNDA 2018) 
Big Data Revolution in Health Care Sector

\subsection{Telemedicine}

Telemedicine has been present on the healthcare services market for over 40 years, but only today, with the arrival of online video conferences, smartphones, wireless devices, and wear able, has it been able to come into full bloom. The term refers to delivery of remote clinical services using technology. It is used for primary consultations and initial diagnosis, remote patient monitoring, and medical education for health professionals. Some more specific uses include telesurgery - doctors can perform operations with the use of robots and high-speed real-time data delivery without physically being in the same location with a patient.

Clinicians use telemedicine to provide personalized treatment plans and prevent hospitalization or re-admission. Such use of big data in healthcare can be linked to the use of predictive analytics as seen previously. It allows clinicians to predict acute medical events in advance and prevent deterioration of patient's conditions.

\subsection{Big Data Prevents unnecessary ER Visits}

Saving time, money and energy using big data analytics in healthcare is necessary. What if we told you that over the course of 3 years, one woman visited the ER more than 900 times? That situation is a reality in Oakland, California, where a woman who suffers from mental illness and substance abuse went to a variety of local hospitals on an almost daily basis. In order to prevent future situations like this from happening, Alameda county hospitals came together to create a program called PreManage ED, which shares patient records between emergency departments.

\section{CONCLUSION}

We may consider Big data as a latest evolution in the field of decision support data management systems. On the other side, the digitalization in health care sector is peak. As we discussed in the paper, there are several opportunities for big data in health care sector. Meanwhile, the technology advancement is rapidly growing on towards the implementation of big data analytics. In near future, there will be widespread implementation of the big data analytics across the health care organization and health care industry. The big data solutions could definitely save millions of life and improve patient services.

\section{REFERENCES}

[1] A. McAfee, E. Brynjolfsson, T. H. Davenport, D. J. Patil, and D. Barton, "Big data: the management revolution", Harvard Business Review, vol. 90, no. 10, pp. 60-68, 2012.

[2] C. Lynch, "Big data: how do your data grow?", Nature, vol. 455, no. 7209, pp. 28-29, 2008. "Insights into the problem of alarm fatigue with physiologic monitor devices: a comprehensive observational study of consecutive intensive care unit patients", PLoS ONE, vol. 9, no. 10, Article IDe110274, 2014.

[3] A. Jacobs, "The pathologies of big data", Communications of the ACM, vol. 52, no. 8, pp. 36-44, 2009.

[4] P. Zikopoulos, C. Eaton, D. deRoos, T. Deutsch, and G. Lapis, Understanding Big Data: Analytics for Enterprise Class Hadoop and Streaming Data, McGraw-Hill Osborne Media, 2011.

[5] J.Manyika,M. Chui, B. Brown et al., Big Data:The Next Frontier for Innovation, Competition, and Productivity,McKinsey Global Institute, 2011.

[6] J. J. Borckardt, M. R. Nash, M. D. Murphy, M. Moore, D. Shaw, and P. O'Neil, "Clinical practice as natural laboratory for psychotherapy research: a guide to case-based time-series analysis”, vol. 63, no. 2, pp. 77-95, 2008.

ISBN: 978-81-936820-0-5

278

Series: AIJR Proceedings

Proceedings DOI: $10.21467 /$ proceedings.1 
[7] L. A. Celi, R.G.Mark, D. J. Stone, and R.A.Montgomery, “Big data' in the intensive care unit: closing the data loop,” American Journal of Respiratory andCritical CareMedicine, vol. 187, no. 11, pp. 1157-1160, 2013.

[8] F. Ritter, T. Boskamp, A. Homeyer et al., "Medical image analysis," IEEE Pulse, vol. 2, no. 6, pp. 60-70, 2011.

[9] J. A. Seibert, "Modalities and data acquisition," in Practical Imaging Informatics, pp. 49-66, Springer, New York, NY, USA, 2010.

[10] B. J. Drew, P. Harris, J. K. Z egre-Hemsey et al., "Insights into the problem of alarm fatigue with physiologic monitor devices: a comprehensive observational study of consecutive intensive care unit patients," PLoS ONE, vol. 9, no. 10, Article IDe110274, 2014.

[11] K. C. GrahamandM. Cvach, "Monitor alarmfatigue: standardizing use of physiological monitors and decreasing nuisance alarms", The American Journal of Critical Care, vol. 19, no. 1, pp. 28-34, 2010.

[12] M. Cvach, "Monitor alarm fatigue: an integrative review", Biomedical Instrumentation \& Technology, vol. 46, no. 4, pp. 268-277, 2012.

[13] J. M. Rothschild, C. P. Landrigan, J. W. Cronin et al., "The Critical Care Safety Study: the incidence and nature of adverse events and serious medical errors in intensive care," Critical Care Medicine, vol. 33, no. 8, pp. 1694-1700, 2005.

[14] P. Carayon and A. P. G"urses, "A human factors engineering conceptual framework of nursing workload and patient safety in intensive care units," Intensive and Critical Care Nursing, vol. 21, no. 5, pp. 284-301, 2005.

[15] P.Carayon, "Human factors of complex sociotechnical systems," Applied Ergonomics, vol. 37, no. 4, pp. 525-535, 2006.

[16] E. S. Lander, L. M. Linton, B. Birren et al., "Initial sequencing and analysis of the human genome", Nature, vol. 409, no. 6822, pp. 860-921, 2001.

[17] R. Drmanac, A. B. Sparks, M. J. Callow et al., "Human genome sequencing using unchained base reads on self-assembling DNA nanoarrays," Science, vol. 327, no. 5961, pp. 78-81, 2010.

[18] T. Caulfield, J. Evans, A.McGuire et al., "Reflections on the cost of 'Low-Cost' whole genome sequencing: framing the health policy debate,” PLoS Biology, vol. 11, no. 11, Article ID e1001699, 2013.

[19] F. E. Dewey, M. E. Grove, C. Pan et al., "Clinical interpretation and implications of whole-genome sequencing," JAMA, vol. 311, no. 10, pp. 1035-1045, 2014.

[20] L. Hood and S. H. Friend, "Predictive, personalized, preventive, participatory (P4) cancer medicine", Nature Reviews Clinical Oncology, vol. 8, no. 3, pp. 184-187, 2011.

[21] L. Hood and M. Flores, "A personal view on systems medicine and the emergence of proactive P4 medicine: predictive, preventive, personalized and participatory", New Biotechnology, vol. 29, no. 6, pp. 613-624, 2012.

[22] L. Hood and N. D. Price, "Demystifying disease, democratizing health care," Science Translational Medicine, vol. 6, no. 225, Article ID 225ed5, 2014.

[23] R.Chen, G. I. Mias, J. Li-Pook-Than et al., "Personal omics profiling reveals dynamic molecular and medical phenotypes," Cell, vol. 148, no. 6, pp. 1293-1307, 2012.

[24] G. H. Fernald, E. Capriotti, R. Daneshjou, K. J. Karczewski, and R. B. Altman, "Bioinformatics challenges for personalized medicine," Bioinformatics, vol. 27, no. 13, Article ID btr295, pp. 1741-1748, 2011.

[25] P. Khatri, M. Sirota, and A. J. Butte, "Ten years of pathway analysis: current approaches and outstanding challenges," PLoS Computational Biology, vol. 8, no. 2, Article ID e1002375, 2012.

Proceedings of the $3^{\text {rd }}$ National Conference on Image Processing, Computing, Communication, Networking and Data Analytics (NCICCNDA 2018) 